\title{
Approach to the Epidemiology of Drug Interactions in Primary Health Care. The Visible Part of a Dangerous Great Iceberg Growing Rapidly
}

\section{Turabian JL*}

Specialist in Family and Community Medicine, Spain

*Corresponding author: Jose Luis Turabian, Health Center Santa Mari, de Benquerencia Toledo, Spain, Email: jturabianf@hotmail.com

\section{Review Article}

Volume 3 Issue 2

Received Date: July 08, 2019

Published Date: August 23, 2019

DOI: $10.23880 /$ eij-16000126

\section{Abstract}

Drug-drug interaction (DDI) is the modification that the action of a medicine undergoes due to the simultaneous presence of another in the organism. The effects of the DDIs are: 1. the appearance of pharmacological adverse reactions, and 2. the decrease in the effectiveness of the treatment. DDIs in outpatient population may be high because of polypharmacy. DDIs are a real problem in clinical practice, which may partly remain hidden. The DDIs are often predictable and preventable. Most DDI studies focus on rates in hospitalized patients. Less is known of DDIs in outpatients; particularly how the general practitioner could it contribute to DDI management by applying his surveillance systems for identifying high-risk medications. In addition, many DDIs are not described by usual databases. On the other hand, there is wide variance in the clinical relevance of these DDIs. The incidence of DDI increases with the number of drugs used and with age. The prevalence and incidence of clinically observable DDIs is between $5-10 \%$ and up to $25 \%$ of patients on pharmacological treatment, and potential DDIs is at least three to five times higher (from $15 \%$ to $50 \%$ ), and even a nearby figure to $100 \%$ in geriatric patients on pharmacological treatment. Pharmacodynamic DDIs are more prevalent $(80 \%)$ than pharmacokinetic. However, the incidence of potentially serious DDIs is relatively low (perhaps less than 1\%) among ambulatory patients. However, the absolute number of patients involved is high, its serious potential risks, and its tendency is to increase rapidly. The clinically recognizable IDDs are like the visible part of an iceberg, where it only stands out between a third or fifth of its total volume, while the rest is "submerged" (potential DDIs and not clinically detected DDIs), which poses a serious danger to the treatment of patients.

Keywords: Drug Interactions; Drug-Related Side Effects and Adverse Reactions/epidemiology; Drug Monitoring/methods; Polypharmacy; Practice Patterns; Inappropriate Prescribing/adverse effects; Primary Health Care; General Practice 


\section{Introduction}

Drug-drug interaction (DDI) is, in a strict sense, the modification that the action of a drug undergoes due to the simultaneous presence of another in the organism. This modification is usually translated into a variation of the intensity (increase or decrease) of the usual effect or the appearance of a different effect (subtherapeutic, therapeutic or toxicological) as expected. The ways of manifesting IDDs are: 1) When the pharmacodynamic or toxic action increases, in relation to only one of the components, which is called synergy (additive or potentiation); 2) When it leads to a decrease in the effect which is called antagonism [1]. So, the health risk effects of the DDIs can be classified in three groups: 1 . the appearance of pharmacological adverse reactions; 2 . the decrease in the effectiveness of the treatment; and 3. the masking or modification of disease symptoms

The consideration of the DDIs has focused on its effect as a cause of adverse drug reaction (ADRs), and within those the most easily to suspect by the general practitioner (GP) are the effects of interactions which are the consequence of synergies or physiological antagonisms. For example, with gentamicin (nephrotoxic in relation to the dose), if used together with other potential nephrotoxic drugs can appear signs of renal toxicity, Or tricyclic antidepressants along with antihistamines add the side effects of drowsiness and dry mouth [1]. DDIs arise in numerous different ways, involving pharmacokinetic or pharmacodynamic mechanisms. DDIs are a major cause of morbidity and mortality. DDIs research includes the study of different aspects of drug interactions, from in vitro pharmacology, which deals with drug interaction mechanisms, to pharmaco-epidemiology, which investigates the effects of DDIs on drug efficacy and adverse drug reactions [2].

DDIs can occur in pharmaceutical phase, pharmacokinetic phase, and in pharmacodynamic phase. In any of these phases, the DDIs can be important. For a long time the actions of drugs on the organism have been studied with interest, but relatively little the actions of drugs among themselves. Practically only galenic interactions or incompatibilities have been studied, that is to say, those that occur within a pharmaceutical preparation, such as physico-chemical reactions, colour changes, precipitation, etc., but there has been little interest about drug-drug effect within the organism. The development of molecular pharmacology systematized the concepts of pharmacological synergy and antagonism, and pharmacokinetics revealed possible interactions at the level of absorption, transport, distribution, metabolization and elimination. Table 1 shows a brief classification of the location of the DDIs $[1,3]$.

\begin{tabular}{|c|c|}
\hline $\begin{array}{l}\text { Location of the Drug-Drug } \\
\text { Interactions }\end{array}$ & Examples \\
\hline 1. Pharmaceutical phase & $\begin{array}{l}\text { The galenic preparation that is administered to the patient. In hospital several } \\
\text { substances are administered via venous via various solutions in perfusion: } \\
\text { antibiotics, vitamins, heparin, corticosteroids, sedatives, etc. }\end{array}$ \\
\hline \multirow{2}{*}{$\begin{array}{l}\text { 2. Pharmacokinetic phase: In } \\
\text { the absorption, distribution, } \\
\text { metabolization and elimination. } \\
\text { The bioavailability -percentage of } \\
\text { effective absorption- can vary } \\
\text { according to chemical factors, } \\
\text { digestive pH, gastric and } \\
\text { intestinal motility }\end{array}$} & $\begin{array}{l}\text { Distribution phase: Most drugs circulate in the blood, bound in a certain } \\
\text { proportion to proteins. Only the free portion is active and can pass to other } \\
\text { compartments. If a drug is displaced (like warfarin) by the action of another, } \\
\text { and its binding to proteins decreases, the concentration increases with the } \\
\text { consequent danger. This risk affects drugs that are combined with proteins in } \\
\text { large proportion. The systems of active transport through membranes can be } \\
\text { modified by certain drugs, favoring or hindering the passage to other }\end{array}$ \\
\hline & $\begin{array}{l}\text { Metabolization phase: Numerous substances can modify the enzymatic } \\
\text { transformation of the drugs, which preferentially settle in the liver, although } \\
\text { drugs are also metabolized in muscle, kidney, lung and other tissues. The } \\
\text { activity of the degrading enzymes can be increased by a large number of usual } \\
\text { drugs. On the contrary, other substances can inhibit your metabolism. The } \\
\text { knowledge of these possible interferences has a great practical interest and } \\
\text { explains many failures or incidents of pharmacological therapy. Here they } \\
\text { should be included the enzyme stimulation or induction, which is associated } \\
\text { with the microsomal synthesis of proteins and cytochrome P450 (for example, } \\
\text { when Postoperative pain might be poorly controlled with prodrug opioids, } \\
\text { which are metabolized by the same hepatic enzyme as selective serotonin } \\
\text { reuptake inhibitors }\end{array}$ \\
\hline
\end{tabular}




\begin{tabular}{|l|l|}
\hline & $\begin{array}{l}\text { Elimination phase: The renal processes of elimination of drugs can suffer } \\
\text { numerous influences. The administration of sodium bicarbonate or sodium } \\
\text { citrate will accelerate the elimination of acidic substances, while the drugs } \\
\text { that produce acidosis (acetylsalicylic acid, antialdosterone diuretics) will } \\
\text { accelerate the elimination of basic medications. }\end{array}$ \\
\hline \multirow{2}{*}{$\begin{array}{l}\text { 3. Pharmacodynamic phase: } \\
\text { at the place where it must act } \\
\text { (receptor area), it can experience } \\
\text { the influence of other drugs, and } \\
\text { increase (synergy) or decrease } \\
\text { its expected effect (antagonism) }\end{array}$} & $\begin{array}{l}\text { Synergy: the achievement of an effect that is the sum of the effects of the } \\
\text { drugs (additive synergy) or greater than the sum of the individual effects of } \\
\text { each drug (enhancing synergy). Examples are combined hypotensors that act } \\
\text { on different receptors, or antipyretic-analgesic, or antibiotic associations (co- } \\
\text { trimoxazole, etc.) }\end{array}$ \\
\cline { 2 - 2 } & $\begin{array}{l}\text { Pharmacodynamic antagonisms: with organic systems or with nervous } \\
\text { mediators, such as central anticholinergic drugs for the treatment of } \\
\text { Parkinson's, antihistamines H1 for the treatment of allergic reactions, or } \\
\text { hormonal contraceptives that are pharmacodynamic antagonists of } \\
\text { hypothalamic regulatory systems }\end{array}$ \\
\hline
\end{tabular}

Table 1: Classification of the Location of the Drug-Drug Interactions.

The increasing clinical importance of drug interactions is justified by the fact that it is currently rare for a hospital patient to receive a single drug. But, it is also currently at the extra hospital level, which is where most of the pharmacological prescriptions are made.

The prevalence of DDIs in outpatient population may be high because of polypharmacy. However, wide variance in the clinical relevance of these interactions has been shown $[4,5]$. This increase in polypharmacy is an indicator of multimorbidity that is also increased by several causes [6]. Polypharmacy, defined as the chronic co-prescription of several drugs, is often the consequence of the application of disease specific guidelines, targeting disease specific goals, to patients with multiple chronic diseases. One common consequence of polypharmacy is the high rate of ADRs, mainly from DDIs. So, DDIs are a significant cause for ADRs. The risk of a DDI in any particular patient increases with the number of co-existing diseases and the number of drugs prescribed [7].

However, there is little information exists on DDIs in outpatient setting, and although it seems that there is a small percentage of outpatient visits with clinically important DDIs, these interactions can have significant implications because of medication-related morbidity and mortality [8].

DDIs are often predictable and preventable, but their prevention and management require systematic service development [9]. Most DDI studies focus on interaction rates in hospitalized patients. Less is known of DDIs in outpatients, particularly how could contribute to DDI management by applying surveillance systems for identifying high-risk medications. On the other hand, there is an underestimation of the importance of DDIs. Thus, it can be thought that DDIs are not a significant problem. Although, the quantification of DDIs in relation to the number of medications prescribed by physicians, dispensed by pharmacists and taken by patients, is not clearly known. In this way, for example, it has been reported that the incidence of hospital admissions by DDIs ranged between 0 and 3\%. However, the existing data are insufficient to allow a significant quantification of specific drugs as usual causes of admissions related to DDIs, and due to the very small number of patients for whom a DDI is believed to be the cause, it is not possible to provide a significant summary of the specific risk factors for admissions by DDIs [10].

In this context, if we take into account that the GP frequently uses several drugs at the same time, knowledge of the phenomena of interaction is fundamental. So, this article, based on a narrative review of the topic, aims to approach some basic data on the epidemiology of DDIs in the outpatient setting.

\section{Discussion}

Currently we know that interaction between different drugs can cause an increase or decrease in the effectiveness of some of them, and the production of unwanted phenomena, from mild to fatal, in addition to modifying the symptoms of diseases in the scenario of patients with multimorbidity and polypharmacy. DDIs are preventable medication errors associated with potentially serious adverse events and death [11].

Drug-drug interactions can also contribute substantially to differences in drug response. Druginduced inhibition of drug metabolising enzymes is usually competitive and causes an increased exposure of the drug whose metabolism is inhibited. This usually results in a higher incidence of adverse drug reactions and an increased severity. Conversely enzyme induction increases the metabolic capacity thereby reducing the 
exposure of a drug with sometimes loss of drug effects (nonresponse). Detailed knowledge of the causative mechanisms will help detecting and avoiding severe adverse drug reactions [12].

The greater the number of drugs that a patient receives, the greater the probability of the appearance of interactions, that modify the activity of one or several of them, and more probability of the appearance of ADRs. although it is absurd to claim the simplification of treatments until use only monosubstances, the most prudent thing is to reduce to the maximum the number of drugs used simultaneously, avoiding the "shot gun therapy" system, thinking wrongly that, the more drugs are used, sooner the patient will be cured.

In the reported data on prevalence and incidence of DDIs, in general have been excluded the drug interactions caused by food (it can decrease the absorption, for example, of amoxicillin or ciprofloxacin), by alcohol (it is inducer or enzyme inhibitor depending on the dose and type of consumption), by grapefruit juice (CYP3A4 inhibitor) or herbalist products such as Hypericum perforatum -St. John's wort (enzyme inducer), which are also relevant in clinical practice [13].

One of the biggest challenges in preventing DDIs is the substantial gap between theory and clinical practice. Despite specific regulatory pathways for drug development and marketing, has not been considered drugs in a holistic way. Drugs have a network of effects that go well beyond a single specific drug target, particularly in patients with multimorbidity. Unwanted effects that are severe and unexpected can surface only during post-marketing surveillance, after many patients have potentially been harmed.

It has to keep in mind that older patients are particularly vulnerable. Prevalence of multimorbidity increases with age, along with the prevalence of polypharmacy. Advancing age is accompanied by physiological changes in the pharmacokinetics and pharmacodynamics of several drugs that might increase the risk of DDIs. Further, older people are underrepresented in guidelines and in the studies on which these guidelines are based. Thus, it is admitted that nearly half of the geriatric outpatients who were taking more than one drug were candidates for DDIs [4].

\section{Incidence and prevalence of DDIs}

It refers to the clinically detectable DDIs, that is, that could lead to measurable patient harm, taking into account the patient's individual clinical profile. Drug interactions could account for $1 \%$ of hospitalizations in the general population and $2-5 \%$ of hospital admissions in the elderly [14]. In every stratum of the population, under-reporting of adverse events from DDIs means it has little idea of their true prevalence and clinical impact.

DDIs arise in numerous different ways, involving pharmacokinetic or pharmacodynamic mechanisms. Some combined medications may result in undesired pharmacodynamic or pharmacokinetic interactions, leading to under-treatment or harmful effects. DDIs are an important cause of ADRs. Primary health care professionals are often unaware of the clinical risks of certain drug combinations [15].

In any case, the current data, which are undoubtedly well below the actual figures, show that pharmacodynamic DDIs are more prevalent (80\%) than pharmacokinetic. Pharmacodynamic DDIs mainly occur with drugs used to treat psychiatric/seizure/sleep disorders (70\%) and pain/migraine (60\%). Pharmacokinetic DDIs mainly occur with drugs used to treat psychiatric/seizure/sleep disorders (50\%), cardiovascular diseases (50\%), and infectious diseases (30\%) [16]. It has also been reported that the most involved drug classes in DDIs are beta blockers, angiotensin-converting-enzyme inhibitors (ACEIs), diuretic agents, and non-steroidal anti-inflammatory drugs (NSAIDs) [17].

So, DDIs could be a real problem in clinical practice (not only theoretically). Since DDIs are an important cause of ADRs [18] and it can be admitted that ADRs occur in approximately $20-25 \%$ of the patients under pharmacological treatment [4,18-20].

One-quarter of these patients could have possible adverse events or diminished treatment effectiveness that may be at least partly caused by these DDIs [4]. Consequently, it can be thought that approximately a minimum of $5 \%-10 \%$ of patients receiving pharmacological treatment have IDD. It must be taken into account that this figure refers to clinically observable DDIs.

Although different authors report similar figures: the $5 \%-10 \%$ of patients receiving pharmacological treatment has IDD. And, the incidence of ADRs (and IDDs) increases with the number of drugs used, being approximately $20 \%$ in patients taking 2 drugs, and $80 \%$ in those taking 6 or more; 7\% of ADRs were due to DDIs. The main adverse symptom of DDI is CNS depression, followed by hemorrhages, severe hypotension and super infection in antibiotics [1, 21].

However, other authors report higher numbers of DDIs identified: $26 \%$. These authors also communicate that no difference between men and women regarding the number of DDIs identified per prescription was 
found. In these studies, the number of drug per prescription was the biggest predictor of the DDIs [22]. But other studies have reported that prescriptions of female patients showed significantly higher number of DDIs [23]. The classes of agents exhibiting gender-based variation in pharmaceutical efficacy and toxicity include anaesthetics, HIV-1 therapies and antiarrhythmic drugs. Further, body weight differences are often cited as a reason for differences in drug pharmacokinetics and subsequent toxicity. However, some studies accounted for these factors and still found significance suggesting that dosage versus body weight does not explain the outcome [24].

\section{Potential vs. Clinically Detected DDIs}

In any case, regarding the frequency or prevalence of the DDIs, it should be taken into account that the frequency of "potential" drug interactions, identified in the records of patients, has been reported to be $30 \%$, with a level similar to the admitted of documented ADRs of $20 \%$ [25]. For example, it has been communicated that prevalence of potentially critical DDIs in outpatients treated with a statin is $7 \%$, mainly due to pharmacodynamic mechanisms. The number of drugs per patient and a diagnosis of arrhythmia or heart failure were identified as risk factors for DDIs [26].

It has been established that approximately a quarter of outpatients visiting a medical center have potential DDI (25\%). There are independent increases in the potential DDI per person in association with aging and the increase in the number of prescription drugs. In addition, there is a significant interaction between these two factors: the effect of aging on the prevalence of potential IDDs increased as the number of prescription drugs increased [27].

So, potential DDIs are identified in 20-30\% of prescriptions. This number of potential DDIs is much higher than the $5-10 \%$ given as the most frequent number and even higher than $25 \%$ of some authors, of the DDIs observed clinically. Clinically significant interaction alerts categorized as FASS classes D (most severe, should be avoided) and C (clinically significant but controllable) are associated with $0.5-2 \%$ and $7 \%$ of the prescriptions, respectively [9].

Major DDIs are identified in 2\% of all prescriptions and represent $10 \%$ of all DDIs detected, whereas moderate DDIs were identified in nearly $20 \%$ of all prescriptions and represented $90 \%$ of all DDIs detected. The rate of DDIs increased with prescription size [28]. Again, these data strongly suggest DDIs could be a real problem in clinical practice, showing that more than one in five patients $(20 \%)$ exposed to a potential DDI experienced a related ADR [18]. Since the reported
ADRs can have a frequency of $5 \%-25 \%$ of patients under pharmacological treatment, it can be inferred that five times more patients are presenting potential DDIs (25\%-100\%).

That is, potential IDDs far outweigh the clinically detected DDIs; the data seem to indicate that, at least, the potential IDDs are found with a frequency three or five times greater than the clinically identified DDIs. Even though the potential for an adverse interaction to occur is often theoretical, and clinically important adverse events occur only in the presence of specific risk factors [15]. Thus, in short, it has been estimated that prevalence of potential DDIs is high, whereas clinically significant DDIs occur a smaller proportion. As always, exposing patients to a greater number of prescription drugs, especially three or more, is proved to be a significant predictor of DDIs [29].

\section{DDIs in geriatric patients}

It has been communicated what prevalence of potentially inappropriate drugs to be avoided among older adults was $60 \%$,where $40 \%$ of the older adults population were prescribed one potentially inappropriate drug, 14\% two potentially inappropriate drugs, and $3 \%$ were on three or more potentially inappropriate drugs. The most commonly prescribed potentially inappropriate drugs were gastrointestinal agents (40\%) and endocrine agents (35\%). Polypharmacy and existence of certain chronic comorbidities are associated with high risk of potentially inappropriate drugs use among older patients [30].

So, it is a fact admitted that elderly patients are at high risk from drug-drug interactions (DDIs). Thus, the situation of elderly patients is special: The overall prevalence of DDIs of all categories in geriatric patients at the ambulatory care has been reported as high as $90 \%$ [31]. However, the incidence of potentially serious DDIs is relatively low (less than 1\%) among ambulatory patients [32].

Anyway, DDIs significantly contribute to the onset of ADRs in older adults [33]. It has been studied the pharmacoepidemiologic of potential drug-drug interactions in geriatric patients in the outpatient and it has been communicated an overall rate of potential DDIs was nearby $30 \%$, with a maximal value of $60 \%$ at the Department of Psychiatry. The rate of the most potentially significant interactions was $3 \%$, being the highest in the Department of Medicine (6\%) [31].

Other authors also communicate that the incidence of DDI-related ADRs in elderly outpatients is high; most events present important clinical consequences and are 
preventable or ameliorable. In this way, it has been communicated the incidence of DDI-related ADRs is $6 \%$. Warfarin was the most commonly involved drug (37\% cases), followed by acetylsalicylic acid (17\%), digoxin (17\%), and spironolactone (17\%). Gastrointestinal bleeding occurred in 37\% of the DDI-related ADR cases, followed by hyperkalemia (17\%) and myopathy (13\%). The multiple logistic regression showed that age $\geq 80$ years (odds ratio -OR- 4.4), a Charlson comorbidity index $\geq 4$ (OR 1.3), consumption of five or more drugs (OR 2.7), and the use of warfarin (OR 1.7) were, in a statistically significant way, associated with the occurrence of DDI-related ADRs. With regard to severity, approximately $40 \%$ of the DDI-related ADRs detected necessitated hospital admission. All DDIrelated ADRs could have been avoided (87\% were ameliorable and $13 \%$ were preventable). The incidence of ADRs not related to DDIs was of $10 \%$ [34].

It has also been published that prevalence of DDIs in older patients was of 33\%. It was detected 1\% A class, $12 \% \mathrm{~B}, 78 \% \mathrm{C}, 9 \% \mathrm{D}$, and $1 \% \mathrm{X}$, according risk rating category of DDIs among the prescriptions. Prescriptions of patients aged between 65 and 70 years showed significantly higher number of DDIs. The frequency of DDIs increased both with the number of drugs and combined preparations per prescription. Acetylsalicylic acid and salbutamol were the most frequently prescribed drugs contributing to clinically important DDIs. Additionally, acetylsalicylic acid and escitalopram, which interact with each other, were found on the list of Beers criteria. The most predicted clinical outcomes of DDIs were increase in therapeutic efficacy and adverse/toxic reactions [23].

\section{Following recommendations for prescription in clinical guidelines would result in several potentially serious drug interactions}

To following the recommendations for prescription in clinical guidelines (NICE clinical guidelines for type 2 diabetes, heart failure, and depression) would result in several potentially serious drug interactions: 32 potentially serious drug-disease interactions between drugs recommended in the guideline for type 2 diabetes and the 11 other conditions compared with 6 for drugs recommended in the guideline for depression and 10 for drugs recommended in the guideline for heart failure. Of these drug-disease interactions $84 \%$ in the type 2 diabetes guideline and all of those in the two other guidelines were between the recommended drug and chronic kidney disease. Few of these DDIs are highlighted in the usual guidelines [35].

In this study, more potentially serious DDIs were identified between drugs recommended by guidelines for each of the 3 index conditions (type 2 diabetes, heart failure, and depression) and drugs recommended by the guidelines for the other 9 conditions commonly comorbid (atrial fibrillation, osteoarthritis, chronic obstructive pulmonary disease, hypertension, secondary prevention after myocardial infarction, dementia, rheumatoid arthritis, chronic kidney disease, and neuropathic pain) with the three index conditions: 133 DDIs for drugs recommended in the type 2 diabetes guideline, 89 DDIs for depression, and 111 DDIs for heart failure. Few of these drug-disease or drug-drug interactions were highlighted in the guidelines for the three index conditions [35].

In consequence, guideline developers could consider a more systematic approach regarding the potential for DDIs, based on epidemiological knowledge of the comorbidities of people with the disease the guideline is focused on, and should particularly consider whether chronic kidney disease is common in the target population. In contrast, potentially serious drug-drug interactions between recommended drugs for different conditions were common. The extensive number of potentially serious interactions requires innovative interactive approaches to the production and dissemination of guidelines to allow clinicians and patients with multimorbidity to make informed decisions about drug selection [35].

\section{Drugs can also interact with genes}

Drugs can also interact with genes, and we have barely keeping in mind on the potential for an additive effect between drug-drug interactions and genetic factors. Clopidogrel is a good example of a drug with a genetically variable therapeutic effect [36]. If a genetically poor metaboliser (with lower bioavailability of active drug) is co-prescribed a proton pump inhibitor, which further reduces the therapeutic effect of clopidogrel, vascular risk increases in an unpredictable way [7]. When compared with DDIs alone, drug-gene interactions and drug-drug-gene interactions increased the total number of potentially clinically significant interactions by $50 \%$ [37].

\section{Identification of the DDIs by the general practitioner}

DDIs are often avoidable and, if undetected, can lead to patient harm. When considering pharmacological interactions, the most easily to suspect by the practical physician are the interactions of effects, which are the consequence of physiological synergies or antagonisms.

European Medicines Agency has already suggested that spontaneous reports of adverse events can be used to identify patterns of drug-disease and DDIs that were not apparent before authorization of the drug. Better education for both patients and doctors about the 
importance of reporting these events is urgently required [7]. But, the surveillance systems are based on different criteria (for example, the FASS database, which classifies the DDI into four classes (A-D) according to their clinical importance, etc.). So there is a lack of systematization and standardization of diagnostic and classification criteria [9].

In any case, the existing studies validates that spontaneous reporting, despite its limitations, can be an important resource for detecting ADRs associated with the concomitant use of interacting drugs [18].

The many types of data sources available for pharmacovigilance research include drug and disease registries, insurance claims and electronic health records databases. The increase in population coverage of available electronic health records data along with increasing numbers of linked datasets can be considered as sources of 'big data'. Increasingly, pharmacoepidemiological studies using this wider range of data sources (including electronic health records databases) are playing a key role in pharmacovigilance activities [37].

The potential interactions can be identified by general practitioner through clinical evaluation [4]. But, many of the DDIs are not described yet by databases [4]. Potential IDDs in outpatients can be reduced by minimizing the number of prescription drugs after careful consideration of their benefits and risks, particularly in older patients [27].

Prediction of DDIs in elderly patients will provide better prescribing and drug safety. Use of nonsteroidal anti-inflammatory agents, selective serotonin reuptake inhibitors, and beta-2 adrenergic receptor agonists should be closely monitored [23]. As more than one in five patients exposed to a potential DDI experienced a related ADR [18], it is recommend that clinicians avoid prescribing potentially inappropriate medications for older adults whenever feasible [38].

The tools allowing identifying co-prescriptions atrisk for interactions appear difficult to use in clinical practice. Many computerized systems have integrated drug safety alerts. Several studies have shown the effectiveness of computers in the early detection of DDI. But, a correct risk-benefit assessment by the prescribing physician is essential, together with a careful clinical, physiological and biochemical monitoring of the patients [15].

It has been described that drug safety alerts in computerized systems are cancelled by clinicians in $50 \%$ to $100 \%$ of cases. The cancellation of alerts can often be justified and adverse drug events due to cancelled alerts cannot always be prevented. A distinction must be made between appropriate and useful alerts. The warning system can contain conditions that produce errors, such as low specificity, low sensitivity, unclear information content, unnecessary interruptions in workflow and unsafe and inefficient handling. This can result in active faults of the doctor, such as ignoring alerts, misinterpretations and incorrect handling. Efforts to improve patient safety by increasing the correct handling of drug safety alerts should focus on the production conditions of errors in the software and the organization [39].

\section{Conclusion}

There is still no clear evidence of the incidence and prevalence of actual DDIs. Standardisation of DDI definitions and research methods are required to allow meaningful prevalence rates to be obtained and compared. Studies that go further than measuring DDIs are critically needed to determine the impact of DDIs on patient safety. More extensive research is needed to identify and minimize factors associated with incidence of DDIs, and to evaluate the effects of preventive interventions especially those that utilize information technology. However, if it can be categorically said DDIs are a real problem in clinical practice, and it is known that more than one in five patients exposed to a potential DDI experienced a related ADR.

Prevalence and incidence of clinically observable DDIs is between $5-10 \%$, according to the most conservative calculations; and even the 25\%. Further, it can be inferred that five times more of the data of clinically observable DDIs, are the patients who present potential DDIs (between 25\% -50\%, and reaching 100\% in patients with polypharmacy).

Thus, the incidence y prevalence of actual clinical DDIs are consistently lower than that of potential DDIs. However, the absolute number of patients involved is high or very high, representing a significant proportion of adverse drug reactions. The importance of risk factors such as age, polypharmacy and genetic polymorphisms must be also evaluated.

The clinically recognizable IDDs are like the visible part of an iceberg, where it only stands out between a third or fifth of its total volume, while the rest is "submerged" (potential DDIs or not clinically appreciated DDIs) (Figure 1). This occurs both due to the difficulty of clinical observation, the difficulties of detecting potential interactions and the difficulties of classification. In addition, as in icebergs, its production is related to the flow rate: the growing increase in multimorbidity and polypharmacy, the aging of the population, and the indiscriminate application of 
guidelines that add drugs to various health problems. All these characteristics make the iceberg of DDIs a great danger for the patient's navigation through the health system.

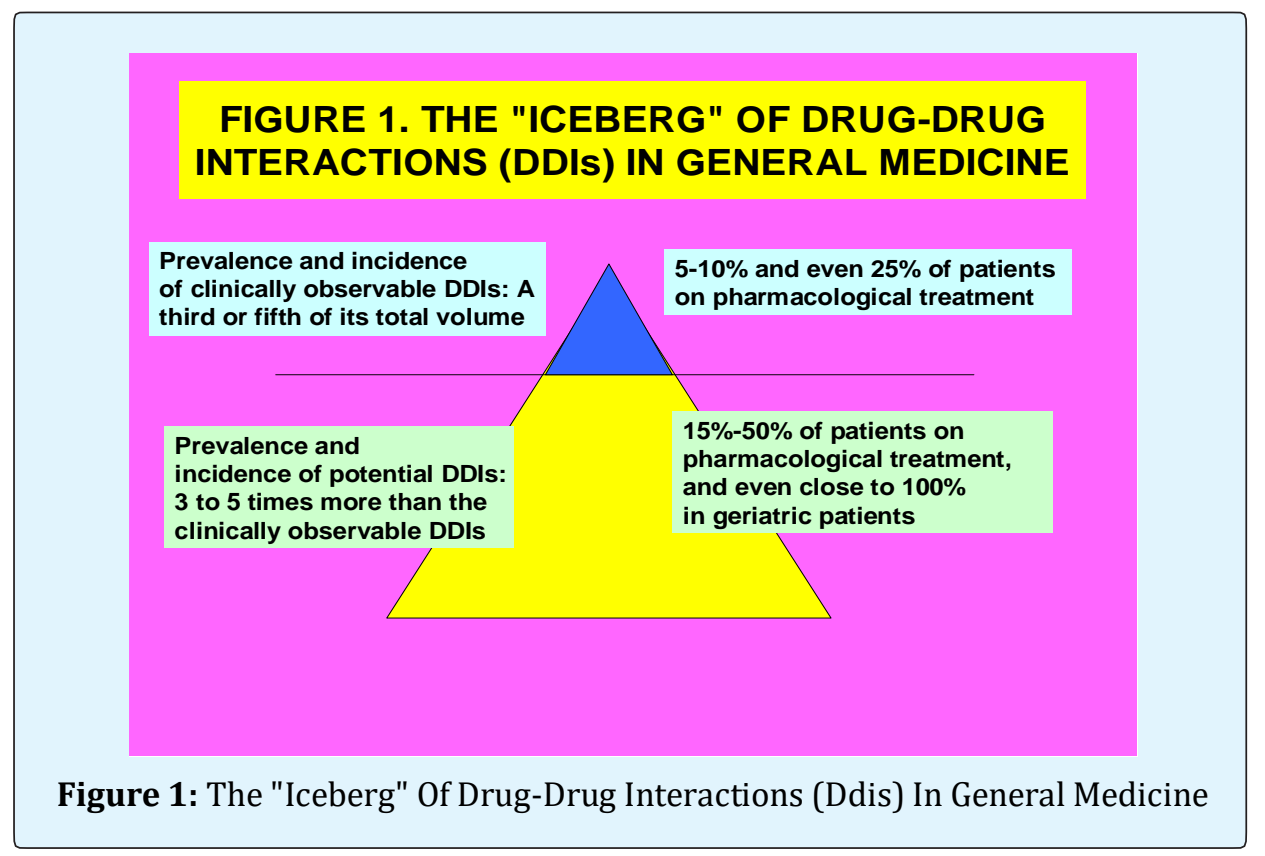

\section{References}

1. Aínsa JLB, Miquel JAS (1980) Reacciones adversas de los medicamentos y enfermedades yatrogénas. Barcelona: Ediciones Toray, S.A.

2. Kolchinsky A, Lourenço A, Li L, Rocha LM (2013) Evaluation of linear classifiers on articles containing pharmacokinetic evidence of drug-drug interactions. Pac Symp Biocomput pp: 409-420.

3. Parthipan A, Banerjee I, Humphreys K, Asch SM, Curtin C, et al. (2019) Predicting inadequate postoperative pain management in depressed patients: A machine learning approach 14(2): e0210575.

4. Tulner LR, Frankfort SV, Gijsen GJ, van Campen JP, Koks CH, et al. (2008) Drug-drug interactions in a geriatric outpatient cohort: prevalence and relevance. Drugs Aging 25(4):343-355.

5. Tulner LR, Kuper IM, Frankfort SV, van Campen JP, Koks CH, et al. (2009) Discrepancies in reported drug use in geriatric outpatients: relevance to adverse events and drug-drug interactions. Am J Geriatr Pharmacother 7(2): 93-104.

6. Turabian JL, Pérez Franco B (2015) Helping "Dr. Theseus" leave the labyrinth of multiple drug interactions. BMJ 350: h1059.
7. Marengoni A, Onder G (2015) Guidelines, polypharmacy, and drug-drug interactions in patients with multimorbidity. BMJ 350: h1059.

8. Aparasu R, Baer R, Aparasu A (2007) Clinically important potential drug-drug interactions in outpatient settings. Res Social Adm Pharm 3(4): 426-437.

9. Toivo TM, Mikkola JA, Laine K, Airaksinen M (2016) Identifying high risk medications causing potential drug-drug interactions in outpatients: A prescription database study based on an online surveillance system. Res Social Adm Pharm 12(4): 559-568.

10. Jankel CA, Fitterman LK (1993) Epidemiology of drug-drug interactions as a cause of hospital admissions. Drug Saf 9(1): 51-59.

11. Gagne JJ, Maio V, Rabinowitz C (2008) Prevalence and predictors of potential drug-drug interactions in Regione Emilia-Romagna, Italy. J Clin Pharm Ther 33(2): 141-151.

12. Mikus G (2011) Introduction to adverse drug reactions and drug-drug interaction. Article in German. Ther Umsch 68(1): 3-9.

13. Morales-Olivas FJ, Estañ L (2006) [Drug-drug interactions. An update. Article in Spanish. Med Clin (Barc) 127(7): 269-275. 
14. Létinier L, Cossin S, Mansiaux Y, Arnaud M, Salvo F, Bezin J, et al. (2019) Risk of Drug-Drug Interactions in Out-Hospital Drug Dispensings in France: Results From the DRUG-Drug Interaction Prevalence Study. Front Pharmacol 10: 265.

15. Magro L, Moretti U, Leone R (2012) Epidemiology and characteristics of adverse drug reactions caused by drug-drug interactions. Expert Opin Drug Saf 11(1): 83-94.

16. Nelson SD, LaFleur J, Hunter E, Archer M, Steinvoort C, et al. (2016) Identifying and Communicating Clinically Meaningful Drug-Drug Interactions. J Pharm Pract 29(2): 110-115.

17. Nabovati E, Vakili-Arki $\mathrm{H}$, Taherzadeh $\mathrm{Z}$, Hasibian MR, Abu-Hanna A, et al. (2014) Drug-drug interactions in inpatient and outpatient settings in Iran: a systematic review of the literature. Daru 22: 52.

18. Leone R, Magro L, Moretti U, Cutroneo P, Moschini $M$, et al. (2010) Identifying adverse drug reactions associated with drug-drug interactions: data mining of a spontaneous reporting database in Italy. Drug Saf 33(8): 667-675.

19. Turabian JL (2019) The role of general practitioner in the study of adverse drug reactions epidemiology in ambulatory care setting. Arch Community Med Public Health 5(1): 003-007.

20. Gholami K, Ziaie S, Shalviri G (2008) Adverse drug reactions induced by cardiovascular drugs in outpatients. Pharm Pract 6(1): 51-55.

21. Ministerio de Sanidad y Consumo. Informes, estudios e investigación (2008) Estudio sobre la seguridad de los pacientes en atención primaria de salud (APEAS) Article in Spanish.

22. Van Heerden JA, Burger JR, Gerber JJ, VlahovićPalčevski V (2018) Prevalence of potentially serious drug-drug interactions among South African elderly private health sector patients using the Mimica Matanović/Vlahović-Palčevski protocol. Int J Pharm Pract 26(2): 156-164.

23. Gören Z, J Demirkapu M, Akpinar Acet G, Çali S, Gülçebi Idriz Oğlu M (2017) Potential drug-drug interactions among prescriptionsfor elderly patients in primary health care. Turk J Med Sci 47(1): 47-54.

24. Nicolson TJ, Mellor HR, Roberts RR (2010) Gender differences in drug toxicity. Trends Pharmacol Sci 31(3): 108-114.
25. Schneider JK, Mion LC, Frengley JD (1992) Adverse drug reactions in an elderly outpatient population. Am J Hosp Pharm 49(1): 90-96.

26. Rätz Bravo AE, Tchambaz L, Krähenbuhl-Melcher A, Hess L, Schlienger RG, et al. (2006) Prevalence of potential drug-drug interactions in ambulatory patients with statin therapy. Praxis 95(5): 139-146.

27. Lin CF, Wang CY, Bai CH (2011) Polypharmacy, aging and potential drug-drug interactions in outpatients in Taiwan: a retrospective computerized screening study. Drugs Aging 28(3):219-225.

28. Chatsisvili A, Sapounidis I, Pavlidou G, Zoumpouridou E, Karakousis VA, et al. (2010) Potential drug-drug interactions in prescriptions dispensed in community pharmacies in Greece. Pharm World Sci 32(2): 187-193.

29. Teixeira JJ, Crozatti MT, dos Santos CA, RomanoLieber NS (2012) Potential drug-drug interactions in prescriptions to patients over 45 years of age in primary care, southern Brazil. PLoS One 7(10): e47062.

30. Alhawassi TM, Alatawi A, Alwhaibi M (2019) Prevalence of potentially inappropriate medications use among older adults and risk factors using the 2015 American Geriatrics Society Beers criteria. BMC Geriatrics 19: 154.

31. Aljadani R, Aseeri M (2018) Prevalence of drugdrug interactions in geriatric patients at an ambulatory care pharmacy in a tertiary care teaching hospital. BMC Res Notes 11: 234.

32. Peng CC, Glassman PA, Marks IR, Fowler C, Castiglione B, et al. (2003) Retrospective drug utilization review: incidence of clinically relevant potential drug-drug interactions in a large ambulatory population. J Manag Care Pharm 9(6): 513-522.

33. Marengoni A, Pasina L, Concoreggi C, Martini G, Brognoli F, et al. (2014) Understanding adverse drug reactions in older adults through drug-drug interactions. Eur J Intern Med 25(9): 843-846.

34. Obreli-Neto PR, Nobili A, de Oliveira Baldoni A, Guidoni CM, de Lyra Júnior DP, et al. (2012) Adverse drug reactions caused by drug-drug interactions in elderly outpatients: a prospective cohort study. Eur J Clin Pharmacol 68(12): 16671676. 
35. Dumbreck S, Flynn A, Nairn M, Wilson M, Treweek $S$, et al. (2015) Drug-disease and drug-drug interactions: systematic examination of recommendations in $12 \mathrm{UK}$ national clinical guidelines. BMJ 350:h949.

36. Verbeurgt P, Mamiya T, Oesterheld J (2014) How common are drug and gene interactions? Prevalence in a sample of 1143 patients with CYP2C9, CYP2C19 and CYP2D6 genotyping. Pharmacogenomics 15(5): 655-665.

37. Ghosh RE, Crellin E, Beatty S, Donegan K, Myles P, et al. (2019) How Clinical Practice Research Datalink data are used to support pharmacovigilance. Ther Adv Drug Saf 10: 2042098619854010.

38. Xing XX, Zhu C, Liang HY, Wang $\mathrm{K}$, Chu YQ, et al. (2019) Associations Between Potentially Inappropriate Medications and Adverse Health Outcomes in the Elderly: A Systematic Review and Meta-analysis. Ann Pharmacother 25: 1060028019853069.

39. Van der Sijs H, Aarts J, Vulto A, Berg M (2006) Overriding of drug safety alerts in computerized physician order entry. J Am Med Inform Assoc 13(2): 138-147. 